\title{
Measles, mumps, and rubella vaccination and bowel problems or developmental regression in children with autism: population study
}

\author{
Brent Taylor, Elizabeth Miller, Raghu Lingam, Nick Andrews, Andrea Simmons, Julia Stowe
}

\begin{abstract}
Objectives To investigate whether measles, mumps, and rubella (MMR) vaccination is associated with bowel problems and developmental regression in children with autism, looking for evidence of a "new variant" form of autism.

Design Population study with case note review linked to independently recorded vaccine data.

Setting Five health districts in north east London. Participants 278 children with core autism and 195 with atypical autism, mainly identified from computerised disability registers and born between 1979 and 1998.
\end{abstract}

Main outcome measures Recorded bowel problems lasting at least three months, age of reported regression of the child's development where it was a feature, and relation of these to MMR vaccination. Results The proportion of children with developmental regression (25\% overall) or bowel symptoms $(17 \%)$ did not change significantly ( $\mathrm{P}$ value for trend 0.50 and 0.47 , respectively) during the 20 years from 1979, a period which included the introduction of MMR vaccination in October 1988. No significant difference was found in rates of bowel problems or regression in children who received the MMR vaccine before their parents became concerned about their development (where MMR might have caused or triggered the autism with regression or bowel problem), compared with those who received it only after such concern and those who had not received the MMR vaccine. A possible association between non-specific bowel problems and regression in children with autism was seen but this was unrelated to MMR vaccination.

Conclusions These findings provide no support for an MMR associated "new variant" form of autism with developmental regression and bowel problems, and further evidence against involvement of MMR vaccine in the initiation of autism.

\section{Introduction}

The suggestion that the measles, mumps, and rubella (MMR) vaccine causes or triggers autism centres on the putative existence of "new variant" autism where developmental regression is reported to follow shortly after MMR vaccination, typically accompanied by bowel symptoms. ${ }^{1}$ Epidemiological studies designed to investigate such a causal association have found no correspondence between temporal trends in MMR uptake and the incidence of autism, nor any evidence of clustering of onset of behavioural disturbance, including regression, shortly after vaccination. ${ }^{2-6}$ However, it is now postulated that the onset of MMR induced "regressive autism" with "autistic enterocolitis" may occur after a prolonged induction interval and, in

\section{Definitions}

Childhood autism

Children with symptoms before the age of 3 years that meet the necessary criteria* under each section of the diagnostic triad for autism: communication difficulties, problems with social interaction, and behaviour problems such as stereotyped repetitions

\section{Atypical autism}

Cases with many of the features of childhood autism but not quite meeting the required criteria for that diagnosis, or with atypical features such as onset of symptoms after age 3 years (also known as pervasive developmental disorder not otherwise specified)

\section{Developmental regression}

A documented deterioration in any aspect of development or reported loss of skills, however transient

*ICD-10 (international classification of diseases, 10th revision) and DSMIV (Diagnostic and Statistical Manual of Mental Disorders, 4th ed)

addition, requires the presence of cofactors such as an intercurrent infection, receipt of antibiotics, a history of atopy, a strong family history of autoimmune disease, or MMR or rubella immunisation of the mother shortly before, during, or after pregnancy. ${ }^{7}$ This modified hypothesis could thereby explain the negative findings of the epidemiological studies. Whatever the postulated induction interval or trends in incidence of autism, the present hypothesis requires that the proportion of autistic children with regression and bowel symptoms be higher in children given MMR vaccine before parents became concerned about their child's development and that the pattern of bowel problems and regression in autism should have changed after MMR was introduced.

We have published epidemiological evidence that showed no causal association between MMR vaccination and autism. ${ }^{23}$ We repeated that survey in late 2000 and early 2001 after an interval of two and a half years in five of the original eight study districts to examine further the relation between MMR vaccine and the onset of autism, particularly autism associated with regression, and to collect information on bowel problems in children with autism.

\section{Methods}

We identified children with childhood and atypical autism born between 1979 and 1998 from computerised health registers of children with disabilities in the community and from special school and child psychiatry records, using the same methods and classifications as in our earlier study. ${ }^{23}$ We abstracted information from the clinical notes then linked the information to independent computerised vaccination records (the



舟 
regional interactive child health system, or TotalCare), which were inputted about the time of vaccination. We recorded bowel problems where the history documented they had lasted at least three months. We also recorded the age at onset of concern about the child's development. We recorded regression if there was documented deterioration in any aspect of a child's development or reported loss of skills.

\section{Statistical analysis}

We used single and multivariable logistic regression models to investigate in detail the relation between exposure to MMR vaccine in relation to onset of autism and the presence of bowel symptoms or regression, with adjustment for potential confounding factors-namely sex, year of birth, district, age at parental concern, and type of autism. We show only single variable results because adjustment for potential confounders did not substantially affect the results.

\section{Results}

We identified 278 children with childhood autism and 195 with atypical autism. Of these 473 children, 81 $(17 \%)$ were reported to have associated bowel problems: constipation (42 children), constipation and diarrhoea (7), diarrhoea (19), food allergy (7), non-specific colitis with ileal-lymphoid-nodular hyperplasia (2), and other (4). The proportion with bowel symptoms was similar in those with childhood and with atypical autism: 49 of $278(18 \%)$ and 32 of 195 $(16 \%)$, respectively $(\mathrm{P}=0.73)$. Regression of the child's development was reported by parents in $118(25 \%)$ of the 469 children where developmental information was recorded. Apparent loss of speech or language skills or change in behaviour were the major forms of regression identified. The proportion with regression was similar in those with childhood and with atypical autism: 43 of 191 (23\%) and 75 of 278 (27\%), respectively $(\mathrm{P}=0.27)$. No significant trends by year of birth were found in the percentages of children with autism who had bowel symptoms (odds ratio $0.98,95 \%$ confidence interval 0.93 to $1.04 ; \mathrm{P}=0.50)$ or with regression $(0.98,0.93$ to $1.03 ; \mathrm{P}=0.47)$ during the 20 years from 1979, a period during which the MMR vaccine was introduced (October 1988) in the United Kingdom (figure). The proportion with regression or bowel problems by year of birth showed a similar pattern for childhood and atypical cases (data not shown).

The single and multivariable logistic regression models confirmed no association between MMR vaccination and regression or bowel symptoms. The table compares children who received the vaccine before their parents became concerned about their development with those who received it only after concern, and those who never received it. No significant difference was found between the proportions in either

Numbers (percentages) of children with autism and bowel problems or developmental regression who had received MMR vaccine before or after their parents became concerned about their development or who had never received vaccine

\begin{tabular}{lcccc} 
& $\begin{array}{c}\text { Vaccine before } \\
\text { parental concern } \\
(\mathbf{n}=257)\end{array}$ & $\begin{array}{c}\text { Vaccine after parental } \\
\text { concern }(\mathbf{n}=\mathbf{1 2 9})\end{array}$ & $\begin{array}{c}\text { No vaccine } \\
(\mathbf{n}=\mathbf{5 7})\end{array}$ & $\begin{array}{c}\text { Overall } \\
(\mathbf{n}=\mathbf{4 4 3})^{\star}\end{array}$ \\
\hline Bowel problems & $50(19)$ & $19(15)$ & $9(16)$ & $78(18)$ \\
\hline Regression & $68(26)$ & $33(26)$ & $17(30)$ & $118(27)$ \\
\hline
\end{tabular}

*Age at parental concern only available for 443 of 473 children.

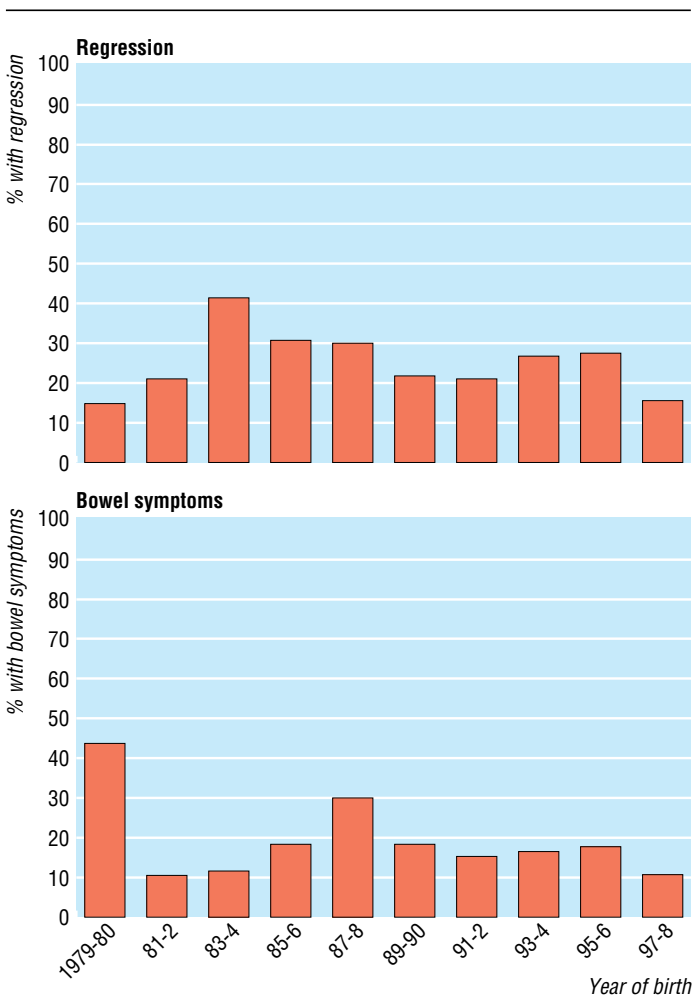

Percentage of children with developmental regression (top) and bowel symptoms (bottom) by year of birth

the single or multivariable models (bowel problems $\mathrm{P}=0.48$; regression $\mathrm{P}=0.83$ ). These analyses were repeated for the 28 cases with bowel problems most likely to reflect the putative disorder "autistic enterocolitis"- that is, food allergy, diarrhoea without constipation, and non-specific colitis with ileallymphoid-nodular hyperplasia. The results showed no evidence of an association with MMR vaccination $(\mathrm{P}=0.57)$, nor a change by year of birth in the proportion with these symptoms (odds ratio 0.98, 0.89 to $1.07 ; \mathrm{P}=0.58$ ).

Although neither bowel problems nor regression was related to MMR vaccination, bowel problems were reported more frequently in children with regression than in those without, 31 of $118(26 \%)$ and 49 of 351 (14\%), respectively $(\mathrm{P}=0.002)$. This relationship between bowel problems and regression did not significantly vary by type of bowel problem $(\mathrm{P}=0.35)$. For the 31 children with both bowel symptoms and regression, there was also no association with MMR vaccination $(\mathrm{P}=0.20)$ and no association with year of birth $(1.01,0.92$ to $1.11 ; \mathrm{P}=0.79)$.

\section{Discussion}

Evidence was lacking to support a "new variant" form of autism, where MMR vaccination is associated with developmental regression and bowel problems. We found no change in the proportion of autistic children with bowel problems or developmental regression over a 20 year period from 1979, a period when MMR vaccination was introduced in the United Kingdom (October 1988). We did find an association between bowel problems and regression in our population, but there was no relation between these factors and MMR 
vaccination. The occurrence of the two symptoms together might reflect particular dietary problems leading to constipation in some children with autism who have regression, or other unknown influences on factors associated with the development and progress of this pattern of autism. Children with autism who have speech loss have more severe learning difficulties several years later than those without such regression. ${ }^{8}$

Bowel symptoms have been reported in an epidemiological study of 96 children with autism, born between 1992 and 1995 in Stafford, England. ${ }^{9}$ All but one of these children had been immunised with MMR vaccine. The study also compared the age at onset of regression and first parental concern with two earlier clinical samples of children, one studied before and the other after the introduction of MMR vaccine (but with no record in those two samples as to whether the individual children had been immunised). No change in the average age of regression or parental concern was noted in the three groups. Bowel problems were recorded only for the most recent sample and, unlike our findings and possibly reflecting smaller numbers, the study found no association between bowel problems and regression. The much larger numbers in our epidemiological study enabled us to look for changes in a population of children with autism over a 20 year period, as well as to allow for possible confounders and, with our complete vaccine linkage, directly to compare regression and bowel symptoms in children who were or were not vaccinated with MMR.

Bowel problems, particularly constipation, occur in many children with various kinds of neurodevelopmental disorder. The percentage we found in our autistic population (17\% overall) is closely similar to the $19 \%$ previously reported..$^{9}$ Those authors supplemented and validated information from the clinical records with questionnaire information directly from the families. That percentages in the two studies are similar provides validation of our method of data collection. Although there have been further reports ${ }^{10}{ }^{11}$ of a specific bowel disorder associated with autism from the group who originally described the possible syndrome, ${ }^{1}$ there is little support for the postulated mechanism involving MMR vaccination. ${ }^{12}$ Many children with autism have unusual diets, often associated with constipation, which might lead to non-specific abnormalities of the bowel. The enteric nervous system is likely to be abnormal in children with autism for whatever the genetic reason for autism turns out to be. ${ }^{12}$

The frequency of regression in autism is uncertain. ${ }^{13}$ One study reviewed published evidence and reported rates varying between 22\% and 50\%. Many children with autism have infantile speech, which usually stops in such children, as in developmentally normal children, before age 18 months. In normal children more communicative speech usually overlaps. The failure of this normal communicative speech to develop in children who have autism, coinciding with the disappearance of infantile vocalisations, may be overinterpreted as regression of speech and language. Our figure of $25 \%$ with developmental regression, although in accord with other studies, is likely to include many such children and is likely to be an overestimate. Regression was found in only $16 \%$ of the contemporary group in the Stafford study, using carefully validated data from a standardised interview for diagnosing autism. ${ }^{914}$ This

\section{What is already known on this topic}

A "new variant" form of autism has been hypothesised, associated with developmental regression and bowel problems and caused or triggered by the MMR vaccination

This postulated association along with media attention has had a major adverse effect on public confidence in the vaccine

Although population studies have shown no association between autism and MMR vaccine it has been further postulated that various environmental or genetic cofactors are required for the effect

\section{What this study adds}

The proportion of children with autism who had developmental regression or bowel problems has not changed over the 20 years from 1979

Neither developmental regression nor bowel problems in children with autism was associated with MMR vaccination

No evidence was found for a "new variant" form of autism

$16 \%$ compared with $18 \%$ in the clinical group who were born between 1954 and 1979 (before MMR vaccination). ${ }^{9}$ These figures support our finding that rates of regression in autism are unchanged over many years.

Our study has the strengths and weaknesses of data based on case notes. Data were not recorded systematically, and there was variability in the level of detail. However, information was available from a variety of sources, including notes from health visitors about the children's early life, and notes from hospitals, child development centres, and other community records such as from schools. Thus a serial record was available for each child, including letters from other centres such as those specialising in autism or paediatric gastroenterology. We assessed the accuracy and consistency of our data collection by duplicating the process independently in 40 cases; interobserver agreement on key variables was over $98 \%$. The review of case notes was carried out independently of the MMR history, which came from a different, independent source (computerised records from the time of vaccination).

A review of each record showed that in 13 children the history given by the parents had changed after the extensive publicity about MMR vaccine and autism. Before the publicity the parents often reported concerns early in their child's life, usually before their first birthday; the current history for the same children recorded symptoms as developing only after MMR vaccination, in some cases shortly after. This bias associated with changes in the history given by the parents necessitates particular care when interpreting the planned selfcontrolled case series analysis of the present dataset. ${ }^{23}$

We thank Pauline Kaye for her help with data analysis and the community paediatricians, child health administrators, and other staff in the districts for their cooperation.

Contributors: BT and EM were responsible for the study design and are guarantors for the paper. BT, RL, and AS were responsible for case ascertainment and the review of case notes. NA undertook the statistical analyses. EM, RL, JS, and BT were responsible for data handling and processing. All authors contributed to writing the paper.

Funding: Department of Health.

Competing interests: None declared. 
1 Wakefield AJ, Murch SH, Anthony A, Linnell J, Casson DM, Malik M, et al. Ileal-lymphoid-nodular hyperplasia, non-specific colitis, and pervasive developmental disorder in children. Lancet 1998;351:637-41.

2 Taylor B, Miller E, Farrington CP, Petropoulos M-C, Favot-Mayaud I, Li J, et al. Autism and measles, mump and rubella vaccine: no epidemiological evidence for a causal association. Lancet 1999;353:2026-9.

3 Farrington CP, Miller E, Taylor B. MMR and autism: further evidence against a causal association. Vaccine 2001;19:3632-5.

4 Kaye JA, Melero-Montes M, Jick H. Mumps, measles and rubella vaccine and the incidence of autism recorded by general practitioners: a time trend analysis. BMJ 2001;322:460-3.

5 Dales L, Hammer S, Smith N. Time trends in autism and MMR immunisation in California. JAMA 2001;285:1183-5.

6 DeWilde S, Carey IM, Richards S, Hilton SR, Cook DG. Do children who become autistic consult more often after MMR vaccination? Brit J Gen Pract 2001:51:226-7.

7 Wakefield AJ. Testimony to the Congress of the United States House of Representatives Committee on Government Reform: hearing on the challenges of autism. Why the increased rates? A one year update. Washington. April 2001. www.house.gov/reform/hearings/healthcare/ 01.04.25/wakefield.htm. [accessed 7 February 2002]

8 Kurita H, Kita M, Miyaka A. A comparative study of development and symptoms among disintegrative psychosis and infantile autism with and without speech loss. J Autism Dev Disord 1992;22:175-88.
9 Fombonne E, Chakrabarti S. No evidence for a new variant of measles-mumps-rubella-induced autism. Pediatrics 2001;108:e58.

10 Wakefield A, Anthony A, Murch S, Thomson M, Montgomery SM, Davies S, et al. Enterocolitis and immunodeficiency in children with developmental disorders. Am J Gastroenterol 2000;95:2285-95.

11 Furlano RI, Anthony A, Day R, Brown A, McGarvey L, Thomson MA, et al. Colonic CD8 and $\gamma \mathrm{d}$ T-cell infiltration with epithelial damage in children with autism. J Pediatr 2001;138:366-72.

12 Gershon M. Testimony to the Congress of the United States House of Representatives Committee on Government Reform: hearing on the challenges of autism. Why the increased rates? A one year update. Washington. April 2001. www.house.gov/reform/hearings/healthcare/ 01.04.25/gershon.htm. [accessed 8 February 2002]

13 Halsey NA, Hyman SL. Conference writing panel. Measles-mumpsrubella vaccine and autistic spectrum disorder: report from the new challenges in childhood immunizations conference convened in Oak Brook, Illinois, Jun 12-13, 2000. Pediatrics 2001;107(5):1-23. www.pediatrics.org/ cgi/content/full/107/5/e84

14 Lord C, Rutter M, Le Couteur A. Autism diagnostic interview-revised: a revised version of a diagnostic interview for caregivers of individuals with possible pervasive developmental disorders. J Autism Dev Disord 1994;24:659-85.

(Accepted 24 January 2002)

\title{
Socioeconomic differences in Swedish children and adolescents injured in road traffic incidents: cross sectional study
}

\author{
Lucie Laflamme, Karin Engström
}

Karolinska

Institutet,

Department of

Public Health

Sciences, Division of

Social Medicine,

SE-171 76

Stockholm, Sweden

Lucie Laflamme

associate professor

Karin Engström

research fellow

Correspondence to:

L Laflamme

lucie.laflamme@

phs.ki.se

BMJ 2002;324:396-7
Traffic related injuries are among the most common causes of death in childhood and in youth. ${ }^{1}$ Young people belonging to a low social class and living in deprived socioeconomic areas are consistently at greater risk than others. ${ }^{2}{ }^{3}$ The extent to which socioeconomic differences in risks from traffic injury vary during childhood and adolescence deserves consideration. ${ }^{45}$ We examined socioeconomic patterning in Swedish children and adolescents injured in road traffic incidents, considering four categories of road users.

\section{Methods and results}

We created a dataset of about 2.2 million children and adolescents (aged 0-19 years) living in Sweden at some time during 1990-4 by linking records from 13 Swedish national registers. We established their sex and year of birth by linking the Swedish population register to the national censuses of 1985 or 1990 or to the medical register of births, according to the person's age.

Subjects were divided into four age groups and allocated to one of four household socioeconomic statuses (table) based on that of the parent with the highest status. The Swedish socioeconomic status is a measure of class, based on occupation and the average level of education required for any particular occupation. Status was attributed to the parents by Statistics Sweden in the Swedish population and housing census of 1990.

We linked the data on sex, year of birth, and socioeconomic status of the young people to five annual national hospital discharge registers (1990-4) and to the national causes of death register. The latter has about $4.5 \%$ of cases lacking information on either E-code (cause of injury) or personal identification number of the injured person. We considered fatal and non-fatal injuries, based on the assumption that the number of lethal injuries did not vary greatly between socioeconomic groups. ${ }^{2}$ We avoided double counting by excluding from the outpatient register any person with the same diagnosis in both registers within two months.

Four diagnostic categories were considered according to ICD-9 (international classification of diseases, ninth revision): pedestrian injuries, bicycle related injuries, injuries as motor vehicle passenger, and injuries as motor vehicle driver (table) (13 772 road traffic injuries in total).

We performed a series of regressions by category of injury diagnosis for each age group to calculate the relative risk of injury according to socioeconomic status. Children of households classified as high or intermediate level salaried employees were used as the reference group. We tested for-but did not find-a possible modification effect of sex of child on socioeconomic patterning; therefore boys and girls were considered together. However, the model used for the later regressions did include the variable for sex of child to test whether boys were at a much greater risk than girls, regardless of socioeconomic status. All analyses were performed using SAS version 6.12.

The relative risks of being injured in a traffic related incident were generally-but not consistently-greater for boys than for girls (table). Socioeconomic differences are negligible in the early years of life (0-4 years) but for all other age groups the relative risks are appreciably higher for children of unskilled workers than for those of high or intermediate level salaried employees. Relative risks are particularly pronounced at 10-14 years of age for non-pedestrians, and at 15-19 years for drivers and riders of motorised vehicles. 